\title{
Síndrome de Munchausen por poderes: presentación de un caso y revisión de la literatura.
}

Munchausen Syndrome by proxi: case presentation and review of the literature.

\author{
Ernesto Landa-Contreras a, María P. Alvites-Ahumada ${ }^{\text {b }}$, José L. Fortes-Álvarez ${ }^{\text {c }}$. \\ ${ }^{a}$ Psiquiatra. ${ }^{b}$ Residente de Psiquiatría. ${ }^{c}$ Psiquiatra y Médico de Familia. ${ }^{a, b y c}$ Hospital Provincial de To- \\ ledo, España.
}

Correspondencia: Ernesto Landa-Contreras (cabiros@gmail.com)

Recibido: 22/12/2013; aceptado: 06/04/2014

RESUMEN: Paciente de 9 años referido para evaluación por múltiples quejas somáticas luego de descartarse patología orgánica en forma exhaustiva. Según su madre tenía una enfermedad rara, mostrándose enfadada porque no se había hospitalizado a su hijo (había sido visto en pediatría, gastroenterología, neurología, urología, etc.). El niño permaneció en todo momento callado, retraído, no permitiendo la madre que sea evaluado solo. La madre acudía repetidamente a su pediatra con su hijo por sintomatología inespecífica, reclamaba que se le hicieran analíticas y pruebas invasivas a su hijo y cuando no se accedía o se le decía que eran normales, se molestaba y reclamaba que se le derive a un especialista. En el artículo se hace una revisión acerca del Síndrome de Munchausen por poderes. PALABRAS CLAVE: Síndrome de Munchausen por poderes.

\begin{abstract}
: 9 year old patient referred for evaluation by multiple somatic complaints after exhaustively ruling out organic pathology. According to his mother he had a rare disease, showing upset because her child had not been hospitalized (he had seen in pediatrics, gastroenterology, neurology, oncology, etc.). The boy remained silent at all times, withdrawn, not allowing the mother to evaluate him alone. The mother repeatedly came to the pediatrician with her child for unspecific symptoms, claiming to do invasive test, and when doctors refused or she was told they were normal, she became upset and claimed to be referred to a specialist. In this article we do a review about Munchausen syndrome by proxy.

KEY WORDS: Munchausen syndrome by proxi.
\end{abstract}

\section{Introducción}

En el DSM IV, el síndrome de Munchausen por poderes (SMP) es parte del Trastorno Facticio (TF) no especificado, caracterizado por un patrón conductual que va desde el reporte de síntomas falsos por parte de los cuidadores del paciente (usualmente la madre) hasta la fabricación de síntomas y perpetración de daño físico (uso de venenos, sofocamiento, infusión de materia fecal, etc.) con el objetivo de asumir el rol de enfermo. Si bien no hay un cuadro clínico característico, si hay patrones de conducta que permiten sospecharlo, lo cual es muy importante ya que de este modo se puede evitar la innecesaria realización de procedimientos que atentan contra la salud del paciente. En el caso de niños, su sospecha es aun más importante ya que al ser considerado un tipo de maltrato, la intervención debe 
ser rápida y oportuna para poder garantizar su seguridad. El objetivo del articulo es hacer una completa revisión de los aspectos más importantes del Síndrome de Munchausen por poderes, a través de su ilustración con un caso clínico.

\section{Caso Clínico}

Paciente varón de 9 años referido por su pediatra para evaluación por múltiples quejas somáticas luego de haberse descartado patología orgánica en forma exhaustiva. La madre refirió que su hijo tenía dificultades para hablar, que había perdido peso, que se mareaba, le dolía el abdomen, que estaba segura que tenía una enfermedad rara y que seguro necesitaba "algunas biopsias". Tenía prácticamente un libro con todos los informes de urgencias, hospitalizaciones y visitas a las consultas de pediatría, gastroenterología, neurología, urología, oncología, etc. En todo momento se mostró enfadada por estar en psiquiatría, según ella, por la incompetencia de los médicos para dar con el diagnóstico de su hijo y recalcando que ya se encargaría de demandar a quien correspondiera por no haberle hospitalizado para estudiarlo a fondo, repitiendo constantemente que su hijo no estaba loco.

El niño permaneció en todo momento callado, retraído, apático respondiendo con monosílabos solo cuando la madre le preguntaba acerca de los síntomas que supuestamente tenía. Fue imposible evaluarlo por separado, ocasionando esto las quejas de la madre que decía que ella no era una ignorante porque había trabajado como secretaria en un centro médico y sabía de lo que hablaba. Cuando se le preguntaba por el niño, decía que todo estaba en los informes.

Dentro de los antecedentes, el paciente vivía con la madre de 35 años (el padre los visitaba cada 3 meses) y 1 hermano de 14 años, no tenía antecedentes de ingresos psiquiátricos ni contacto previo con psiquiatría-psicología. Tanto la gestación como el desarrollo psicomotor fueron normales. A partir de los 6 años comenzó a tener continuas visitas a las urgencias, por sintomatología múltiple e inespecífica (dolor abdominal, mareos, cefaleas, pérdida de conocimiento, etc.), y casi siempre se le daba de alta luego de que el exámen físico y las analíticas fueran normales.

Tuvo 2 ingresos a los 7 y 8 años, por supuesta hematuria y pérdida de conocimiento respectivamente. Durante el primer ingreso tanto el exámen físico, las analíticas y la evolución fueron normales, no volviendo a presentar la supuesta hematuria. Se describió a la madre como problemática, exigiendo la realización de más pruebas antes del alta. Al final le hicieron una urografía y cistoscopía que resultaron también negativas. El segundo ingreso (por pérdida de conocimiento) tuvo el mismo patrón, con exámen, analíticas, CT y RM normales.

Luego de revisar los informes, se les hizo salir para contactar a la pediatra. 
NOTAS CLÍNICAS

Consulta con la pediatra: la pediatra refirió que la madre acudía muchas veces a la consulta con su hijo por sintomatología inespecífica que no cuadraba con ningún diagnóstico, reclamaba que se la hagan analíticas y/o pruebas invasivas a su hijo y que cuando no se accedía o se le decía que eran normales, se enfadaba, reclamando que se le derive a un especialista. Nunca dejaba que se evalúe solo a su hijo y dominaba algunos términos médicos. Durante los ingresos inicialmente se mostraba muy colaboradora con los médicos, quedándose todo el día en el hospital e incluso ofreciéndose a cuidar a otros enfermos, pero su actitud cambiaba cuando se le decía que todo estaba normal o se le daba de alta a su hijo. La madre tenía historia de múltiples quejas somáticas y visitas a las urgencias que remitieron luego del nacimiento de su hijo menor, siempre se había negado a ir al psiquiatra.

Nuevamente se hizo pasar a la madre quien manifestó que ya se tenía que ir porque no tenía el apoyo de nadie, que su marido nunca había cuidado de su hijo y que seguro el ahora necesitaba una tomografía. Se intentó preguntarle más por la historia de su hijo, pero repetía que todo estaba en los informes. Cuando se le pregunto si le podíamos evaluar solo, se enfadó y salió. Se le insistió que era necesario que vuelva a acudir, pero nunca regresó.

\section{Discusión}

En el síndrome de Munchausen por poderes el perpetrador intencionalmente produce o finge síntomas/signos físicos o psicológicos en otra persona que está bajo su cuidado (usualmente un niño). La motivación (como en cualquier facticio) es asumir el rol de enfermo (búsqueda de atención médica) pero a través de otra persona sin haber una ganancia secundaria. Muchos lo consideran como una forma de abuso infantil, ya que puede resultar en la realización de exámenes y procedimientos innecesarios incluyendo cirugías.

En el Reino Unido, su incidencia de 2,8 casos por 100000 en menores de 1 año y 0,5 en mayores de 16 años (1). En USA, se reportan 200 nuevos casos por año con igual frecuencia en hombres y mujeres. En casi el 95\% de los casos el victimario es la madre (2). El inicio de abuso suele ser hacia los 3 años, pasando usualmente 2 años desde que comienzan los síntomas hasta que se hace el diagnóstico y ocurriendo el $70 \%$ de casos de SMP cuando el paciente está hospitalizado. Entre 8-25\% tienen hermanos con el mismo problema (3-4).

Dentro de las teorías que tratan de explicar la motivación de estas personas están el deseo de llamar la atención, el ganar gratificación emocional y reconocimiento al asumir el rol de madre dedicada (mientras que en privado el niño es sujeto de abuso físico/emocional al deliberadamente hacer que finja síntomas) el cual 
es satisfecho al tener alguna relación con el personal sanitario (recibir la atención del médico), etc. (5).

Schreier et al (6) sostiene que lo fundamental en pacientes con SMP es la relación madre-médico, es decir que cuando la atención del médico hacia la madre o niño disminuye, los síntomas empeoran para conseguir ganar mayor atención del médico o reconocimiento como una buena madre (y suplir sus carencias emocionales), o incluso ser vistas como "mejores personas" que los médicos ("adicción al médico").

También se habla de un vínculo patológico madre-niño, asumir el rol de enfermo a través de su supuesto niño enfermo como una forma de pedir ayuda para sus propios problemas. En muchas de estas madres hay una historia de traumas y abusos en su infancia por lo que se ha sugerido que estas personas canalizarían sus inseguridades y síntomas afectivos a través de conductas abusivas hacia sus hijos, para de este modo obtener atención del médico (gratificación emocional) $(5,7)$. Bass (8) encontró historia de TF o Somatomorfo en las madres de estos pacientes y lo identifica como un factor de riesgo.

En cuanto al diagnóstico, la presencia de múltiples síntomas, inexplicables, persistentes o recurrentes que no se pueden explicar con una base médica (discrepancias entre la historia, los hallazgos clínicos y el estado del paciente) o síntomas que no responden al tratamiento habitual y ocurren solo en presencia del victimario, desapareciendo en su ausencia o reapareciendo cuando este es informado que el paciente se está recuperando o va a ser dado de alta, deben alertar respecto de un posible SMP. En cuanto a los patrones de conducta, una madre más preocupada por el personal médico que por la enfermedad de su niño (buscando establecer relaciones con el personal médico para luego pedir más procedimientos diagnósticos y terapéuticos independientemente del malestar que estos le puedan causar al niño, provocando cualquier negativa un cambio de actitud o que se quieran llevar al niño a otro médico o pidan el alta), con experiencia en el campo de la salud (Meadow (9) encontró que $80 \%$ de los victimarios trabajaban en instituciones sanitarias) o con historia de enfermedad psiquiátrica (TF hasta en $25 \%$ de los casos) también debería hacernos pensar en esta posibilidad $(3,10)$.

En cuanto a las víctimas, algunos llegan a pensar que realmente tienen una enfermedad desconocida, otros saben que no es así, pero temen hablar por las represalias de la madre, aprendiendo a tolerar pasivamente los procedimientos médi$\cos (3)$.

Es fundamental en este diagnóstico una evaluación multidisciplinaria, con revisión de historia clínica previa, hospitalizaciones, consultas, historia social y de la familia por las consecuencias médico-legales del mismo.

En cuanto al tratamiento lo primero es asegurar la seguridad de la victima (niño), a través de la intensa supervisión del perpetrador o en algunos casos remo- 
ción temporal o permanente del niño por su seguridad (11). Se debe individualizar cada caso. Usualmente los pacientes requieren psicoterapia prolongada pero suelen ser renuentes, lo mismo se aplica a los cuidadores $(6,10)$

Debido a todo esto el pronóstico no es favorable, habiéndose reportado una mortalidad de $6 \%$ y un $7.3 \%$ de lesiones a largo plazo (4). Muchos de los pacientes terminan con algún tipo de trastorno psiquiátrico (trastorno de estrés postraumático, trastorno oposicionista, depresión, ansiedad, fracaso escolar, trastornos de la personalidad, etc.) (12). Uno de los mayores riesgos es que terminen convirtiéndose en futuros victimarios, es decir que haya una perpetuación del SMP.

\section{BIBLIOGRAFIA}

(1) Denny SJ, Grant CC, Pinnock R. Epidemiology of Munchausen Syndrome by Proxy in N Z. J Pediatrics Child Health 2001;37:240-243.

(2) Feldman KW, Feldman MD, Grady R, Burns MW, McDonald R. Renal and urologic manifestations of pediatric condition falsification/Munchausen by proxy. Pediatr Nephrol 2007;22(6):849-56.

(3) Forsyth B. Munchausen Syndrome by Proxy. Child and Adolescent Psychiatry: A Comprehensive Textbook. $2^{\mathrm{a}}$ ed. Baltimore:Williams \& Wilkins, 2002.

(4) Sheridan MS. The deceit continues: an updated literature review of Munchausen Syndrome by Proxy. Child Abuse Negl 2003;27(4):431-51. 1054.

(5) Forsyth BWC. Munchausen Syndrome by Proxy. Child Adolesc Psychiatry 1996;1048-

(6) Schreier HA, Libow JA. Munchausen by Proxy Syndrome: a modern pediatric challenge. J Pediatrics 1994;125:110-115.

(7) Squires JE, Squires RH. J Munchausen syndrome by proxy: ongoing clinical challenges. Pediatr Gastroenterol Nutr 2010; 51(3):248-53

(8) Bass C, Jones D. Psychopathology of perpetrators of fabricated or induced illness in children: case series.Br J Psychiatry 2011;199(2):113-8.

(9) Meadow R. Munchausen Syndrome by Proxy. Arch Dis Childhood 1982;57:92-98.

(10) Moldavsky M, Stein D. Munchausen Syndrome by Proxy: two cases report and an update of literature. Int J Psychiatry Med 2004;33(4):411-423.

(11) Stirling J. Beyond Munchausen Syndrome by Proxy: Identification and treatment of child abuse in a medical setting. Pediatrics 2007;119:1026-1030.

(12) Bools CN, Neale BA, Meadow SR. Follow up of victims of fabricated illness (Munchausen syndrome by proxy). Arch Dis Child 1993;69(6):625-30. 